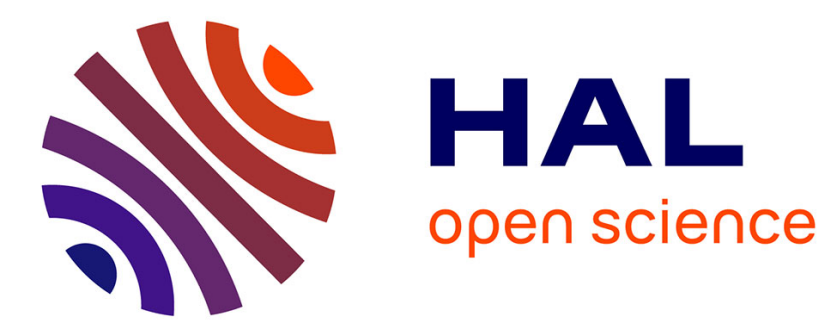

\title{
The freezing transition of monolayer xenon on single crystal graphite
}

\author{
E.D. Specht, R.J. Birgeneau, K.L. d'Amico, D.E. Moncton, S.E. Nagler, P.M.
} Horn

\section{> To cite this version:}

E.D. Specht, R.J. Birgeneau, K.L. d'Amico, D.E. Moncton, S.E. Nagler, et al.. The freezing transition of monolayer xenon on single crystal graphite. Journal de Physique Lettres, 1985, 46 (12), pp.561-567. 10.1051/jphyslet:019850046012056100 . jpa-00232560

\section{HAL Id: jpa-00232560 https://hal.science/jpa-00232560}

Submitted on 1 Jan 1985

HAL is a multi-disciplinary open access archive for the deposit and dissemination of scientific research documents, whether they are published or not. The documents may come from teaching and research institutions in France or abroad, or from public or private research centers.
L'archive ouverte pluridisciplinaire HAL, est destinée au dépôt et à la diffusion de documents scientifiques de niveau recherche, publiés ou non, émanant des établissements d'enseignement et de recherche français ou étrangers, des laboratoires publics ou privés. 
Classification

Physics Abstracts

64.70D $-61.10 \mathrm{~F}-61.25 \mathrm{~B}$

\title{
The freezing transition of monolayer xenon on single crystal graphite
}

\author{
E. D. Specht, R. J. Birgeneau \\ Department of Physics, Massachusetts Institute of Technology, Cambridge, MA 02139, U.S.A. \\ K. L. D’Amico, D. E. Moncton \\ Brookhaven National Laboratory, Upton, NY 11973, U.S.A.
}

S. E. Nagler $\left(^{*}\right)$ and P. M. Horn

IBM T.J. Watson Research Center, Yorktown Heights, NY 10598, U.S.A.

(Reçu le 4 mars 1985, accepté sous forme définitive le 23 avril 1985)

\begin{abstract}
Résumé. - Nous avons utilisé la diffraction des rayons $\mathrm{X}$ de synchrotron pour étudier la transition fluide-solide d'une monocouche de xénon sur un monocristal de graphite. Le système montre une évolution continue d'un fluide orienté vers une phase solide. La longueur de corrélation du fluide dépasse $2000 \AA ̊$ avant que la phase solide soit atteinte. Les pics solides sont un peu élargis anisotropiquement de sorte que le cristal simule une phase hexatique trempée, probablement à cause des impuretés ou des défauts de surface.
\end{abstract}

\begin{abstract}
Synchrotron X-ray diffraction studies of the freezing transition of monolayer xenon on single crystal graphite are reported. The system displays a continuous evolution from an oriented fluid to an incommensurate solid phase. The fluid correlation length reaches at least $2000 \AA$ before the solid phase is entered. The solid peaks are slightly broadened anisotropically such that the crystal simulates a quenched hexatic, presumably because of surface defects or impurities.
\end{abstract}

In spite of a prodigious experimental and theoretical effort, no consensus has emerged as to the nature of freezing in two dimensional (2D) continuous symmetry systems. An elegant theory based on the concept of the successive binding of pairs of disclinations and dislocations suggests that the freezing could occur in two steps : from isotropic fluid to orientationally ordered fluid to solid, where both transitions are predicted to be continuous [1, 2]. In systems which solidify into a triangular lattice the proposed intermediate phase has been labelled " hexatic " [2]. Other theories, however, predict a first order freezing transition with no intermediate hexatic phase, at least in the absence of a substrate [3]. In many cases, experiments show that the freezing transition is indeed first order [4]. However, high resolution synchrotron X-ray scattering measurements for monolayer incommensurate xenon and krypton on graphite provide strong evidence

(*) Present Address : Department of Physics, University of Florida, Gainesville, Florida 32611, U.S.A. 
for a continuous transition from an orientationally ordered fluid to a solid [5-7]. These experiments show that the fluid correlation length reaches at least $200 \AA$ before the solid forms; further, the angular width scales linearly with radial width, consistent with theoretical expectations [6].

In spite of the above experiments, other investigators continue to argue that even for rare gases on graphite the freezing is always first order [8]. Of course, it is impossible to rule out an arbitrarily small first order jump. Nevertheless, it is important to extend the limits of the fluid positional correlation length at freezing and to obtain more accurate information on the orientational fluctuations. In the previous synchrotron X-ray scattering experiments [5-7], the limit on the resolution has been determined by the graphite substrate rather than by the X-ray spectrometer. In order to address this issue we have developed a sample cell which enables us to carry out phase transition studies using a single crystal graphite substrate. Experiments on the rotational transition of monolayer krypton on graphite using this apparatus have been reported previously [9]. The surface coherence length was found to be at least $10,000 \AA$. In this note, we report a study of the freezing of $\sim 0.9$ monolayers of xenon on single crystal graphite. We find that the fluid positional correlations extend to at least $2000 \AA$ before solidification occurs; again the angular width scales linearly with the radial width. Thus, any first order jump must be extraordinarily small.

The experiments were carried out on Beam Line VII-2 at the Stanford Synchrotron Radiation Laboratory (SSRL); beam line and diffractometer hardware were previously described [10]. As discussed in reference [9], we used a natural graphite single crystal which was cleaved in air to produce a wafer approximately $0.03 \mathrm{~mm}$ thick. The sample was mounted in a beryllium cell connected to a UHV gas-handling system and baked out prior to this experiment. Scattering occurred from overlayers on the two cleavage surfaces. Sample heating in situ was accomplished by direct resistive heating to $600^{\circ} \mathrm{C}$. The cell was mounted in a closed cycle refrigerator.

Figures 1 and 2 show, respectively, angular and radial scans through a commensurate kryp-

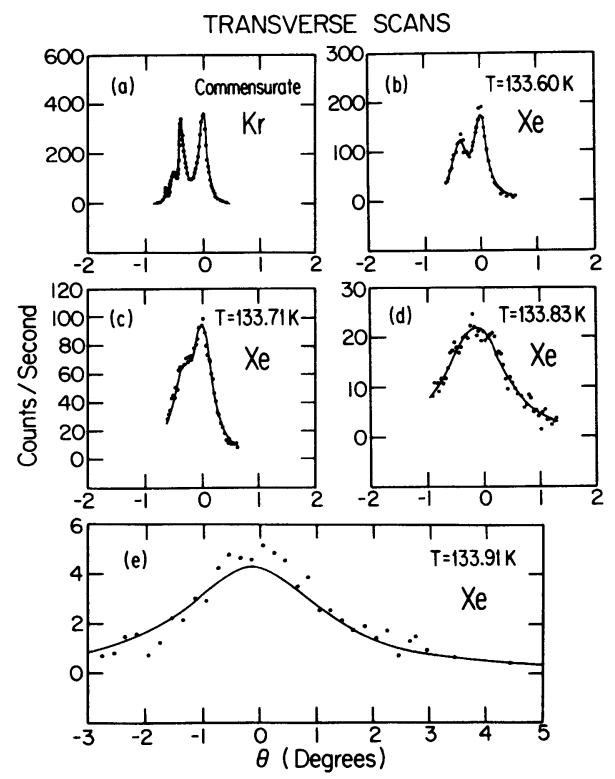

Fig. 1. - (a) Transverse scan through the monolayer krypton $(1,0)$ peak in the commensurate solid phase; the solid line is a least squares fit to a sum of Lorentzians. (b)-(e) Transverse scans through the monolayer xenon $(1,0)$ peak in the solid and fluid phases. The solid lines are the results of fits to a Lorentzian convoluted with the instrumental resolution function; the latter is determined by the substrate mosaic as given by the krypton scan. 


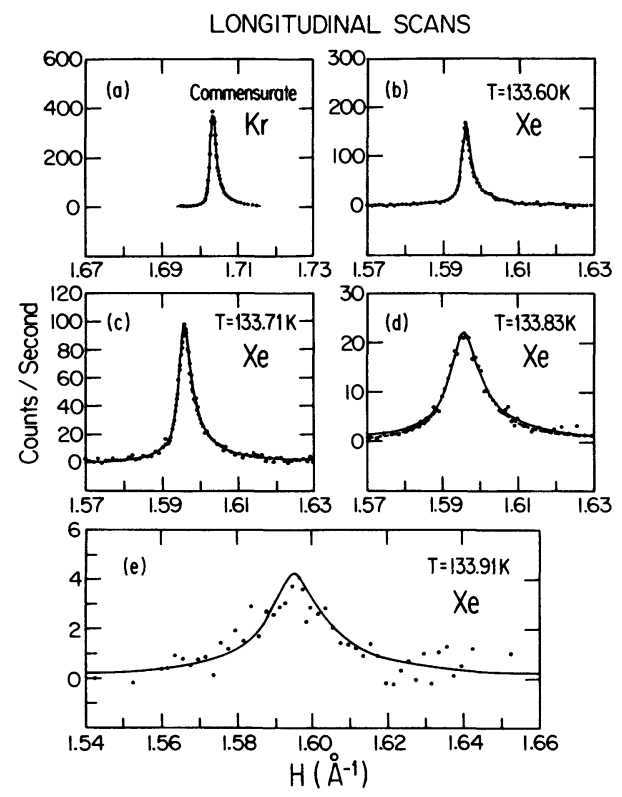

Fig. 2. - (a) Longitudinal scan through the monolayer krypton $(1,0)$ peak in the commensurate solid phase; the solid line is a fit to a sum of Gaussian and Lorentzian [15] convoluted with the vertical mosaic. (b)-(e) Longitudinal scans through the monolayer xenon $(1,0)$ peak in the solid and fluid phases. The solid lines are the results of fits to equations (1) and (2) as discussed in the text; the dashed line in (d) is the best fit for an anisotropic Lorentzian structure factor convoluted with the mosaicity alone.

ton $(1,0)$ peak (panel a) and through the incommensurate xenon $(1,0)$ peak (panels b-e) at a series of temperatures bracketing the freezing transition. For the xenon measurements the gas pressure in the cell was fixed at 2.3 torr; previously Dimon et al. [5] had shown that for $T$ fixed at $134 \mathrm{~K}$, solidification occurs at $P \sim 2$ torr; using the Thomy-Duval definition of point $\mathrm{B}_{1}$ in the isotherms as a complete monolayer [11], Dimon et al. [5] determined that at the point $(P, T)=(2.05 \mathrm{~T}, 134 \mathrm{~K})$, the coverage is 0.88 monolayer. It should be noted, however, that at point $\mathrm{B}_{1}$ the $(1,0) Q$-vector is $\sim 1.63 \AA^{-1}$, compared with $1.594 \AA^{-1}$ at solidification; thus, the coverage in units of the primitive cell area at solidification is closer to 0.92 monolayers.

The commensurate krypton diffraction scans shown in figures 1a and 2a have already been discussed in [9]. In the following, transverse and longitudinal refer respectively to angular and radial scans through the overlayer $(1,0)$ reciprocal lattice position. The transverse scan shows two main peaks together with several small, very sharp sub-peaks; the smaller peaks are as sharp as $0.01^{\circ}$ halfwidth at half maximum (HWHM) while the two main peaks have a $\mathrm{HWHM}$ of $0.07^{\circ}$. The longitudinal scan has a HWHM of $0.0008 \AA^{-1}$, corresponding to a crystallite size of $10,000 \AA$. The best-fit vertical mosaic is $1.5^{\circ}$. It should be emphasized that both the mosaicity and the finite krypton overlayer particle size originate from the substrate. They do not reflect fundamental 2D physics and will improve with yet higher quality graphite single crystal substrates [12]. Xenon diffraction data are given in figures $1 b-1 e$ and $2 b-2 e$ which show representative scans from the freezing point up to the point where the fluid positional correlation length has decreased to $200 \AA$. Graphite and xenon gas scattering have been subtracted, scaled by a variable factor to account for attenuation due to xenon. From scans $1 b$ and $2 b$, as well as other scans at lower temperatures not shown here, it is evident that even in the solid phase the xenon profiles are broader than those for commensurate krypton implying that the incommensurate xenon is less ordered.

In order to analyse these data quantitatively, it is necessary to choose a specific form for the 
fluid structure factor. This is discussed extensively in reference [6]. We have used the following approach. The positional correlations are assumed to give rise to a circular Lorentzian

$$
S_{\mathrm{p}}(\mathbf{Q})=\frac{A}{\kappa^{2}+q_{\mathrm{L}}^{2}+q_{\mathrm{T}}^{2}}
$$

where $\left(q_{\mathrm{L}}, q_{\mathrm{T}}\right)=\mathbf{Q}-\mathbf{G}$ and $\kappa=1 / \xi_{\mathrm{p}}$ is the inverse positional correlation length. In order to include angular effects it is necessary to include the line broadening from orientational fluctuations. We assume that these can be incorporated by convolving equation (1) with a Lorentzian in the transverse direction [6]

$$
f\left(q_{\perp}\right)=\frac{G \Delta \theta / \pi}{(G \Delta \theta)^{2}+q_{\perp}^{2}}
$$

As a consequence, the transverse scan is a Lorentzian with HWHM $\kappa+G \Delta \theta$, so that the orientational fluctuations manifest themselves as an excess width $G \Delta \theta$. The longitudinal function, which may be evaluated exactly, has the form of a Lorentzian for $\left|q_{\perp}\right| \gg G \Delta \theta$ crossing over to the square root of a Lorentzian for $\left|q_{\perp}\right| \ll G \Delta \theta$; here we have implicitly assumed $\kappa<G \Delta \theta$.

We choose as the resolution function the commensurate krypton profile. To evaluate $\kappa$ and $G \Delta \theta, S(\mathbf{Q})$ is convoluted with the system resolution function and fitted in a least squares sense to the measured transverse and longitudinal scans. The solid lines in figures 1 and $2 \mathrm{~b}$-e are the resulting best fit profiles; clearly the model works very well. In order to test for the uniqueness of our model for $S(\mathbf{Q})$, we also fitted the longitudinal scans to a pure Lorentzian and to the square root of a Lorentzian, each convoluted with the instrumental resolution function. The fits to the square root of a Lorentzian are quite poor; however, the results for the pure Lorentzian are indistinguishable from those obtained with the above model ; figure $2 \mathrm{~d}$ illustrates the greatest difference observed. This insensitivity to the excess width $G \Delta \theta$ occurs because the sample mosaicity plays a dominant role in determining the net angular width for most of the data. Specifically, $\Delta \theta$ typically is $0.2^{\circ}$ compared with the $0.33^{\circ}$ separation of the two principal mosaic peaks. Experiments utilizing a substrate with a single sharp peak in the mosaic structure are required to determine the intrinsic form of the structure factor $S(\mathbf{Q})$ [12].

The resulting $\kappa$ and $G \Delta \theta$ are shown in figures 3 and 4. Previous experiments in this temperature range by Dimon et al. [5] using ZYX graphite as a substrate showed that the fluid correlation length reached at least $200 \AA$ before solidification occurred. As shown in figure 3 , fits of these single crystal data to equations (1) and (2) suggest that the positional correlation length evolves smoothly up to $\sim 10,000 \AA$. However, following Heiney et al. [5], in order to provide a more rigorous limit we have explicitly fitted the data to a fluid-solid coexistence model allowing an arbitrary amount of solid. For all scans with $T \geqq 133.7 \mathrm{~K}$, the fits give a negligible solid component. This, in turn, necessitates that the correlation length reaches at least $2000 \AA$ before freezing occurs. Any first order jump must be extraordinarily small.

The excess angular width $G \Delta \theta$ is plotted versus the positional width $\kappa$ in figure 4. Throughout the fluid range, $G \Delta \theta$ appears to scale linearly with $\kappa$ with a ratio of about 3. Nagler et al. [6] have previously found $G \Delta \theta \sim 5 \kappa$ for $\kappa$ varying between $0.1 \AA^{-1}$ and $0.01 \AA^{-1}$. Combining the Nagler et al. [6] data with those in figure 4, we have the surprising result that for fluid correlation lengths covering the range from $\sim 10 \AA$ to $\sim 2000 \AA$ the angular width is nearly a linear function of the inverse positional correlation length.

The theory for the angular width is discussed extensively in reference [6]. We summarize the essential features briefly here. In a well-correlated fluid there are two important lengths, the positional correlation length $\xi_{\mathrm{p}}$ and the orientational length $\xi_{6}$ [2]. At the isotropic fluid-hexatic transition, $\xi_{6}$ diverges. However, even in the isotropic fluid phase, one expects the fluid to res- 


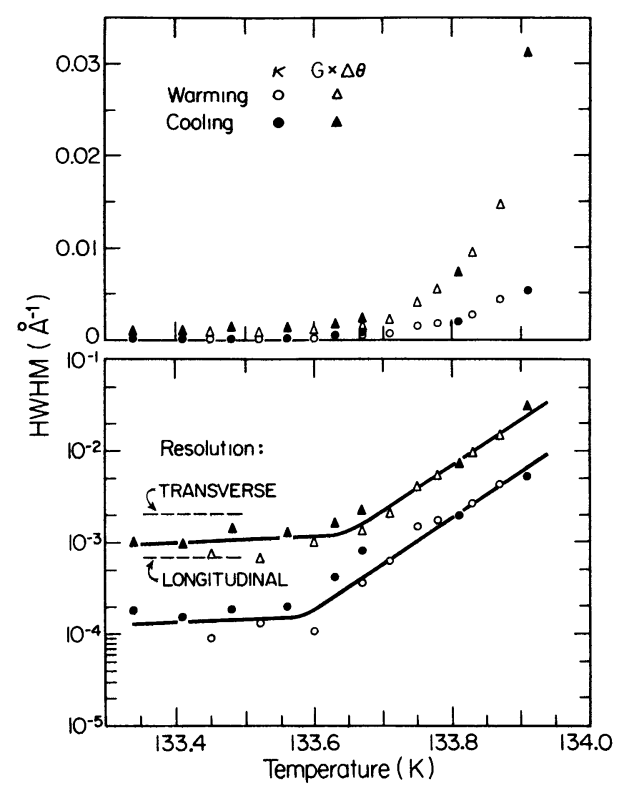

Fig. 3. $-\kappa$ and excess angular width $G \Delta \theta$ versus temperature on linear and logarithmic scales. Dashed lines indicate the width of the resolution function; solid lines are guides to the eye.

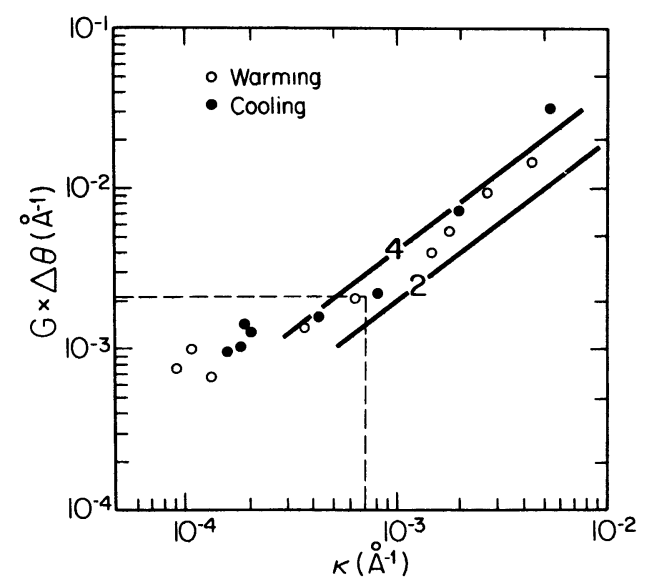

Fig. 4. - Excess angular width $G \Delta \theta$ plotted versus the inverse correlation length $\kappa$; the dashed lines give the resolution limits; the solid lines correspond to $G \Delta \theta=2 \kappa$ and $G \Delta \theta=4 \kappa$, respectively.

pond like a hexatic for fluctuations on length scales between $\xi_{\mathrm{p}}$ and $\xi_{6}$. The Hamiltonian in the hexatic phase is

$$
\mathscr{H}=\frac{K_{\mathrm{A}}}{2} \int(\nabla \theta)^{2} \mathrm{~d}^{2} r+\frac{1}{2} \int h_{6} \theta^{2} \mathrm{~d}^{2} r
$$

with $K_{\mathrm{A}} \sim E_{\mathrm{c}} \xi_{\mathrm{p}}^{2}$, where $E_{\mathrm{c}}$ is the dislocation core energy [2]. Here $h_{6}$ is the substrate orienting field aligning the overlayer $(1,0)$ axis with respect to the substrate $(1,1,0)$ axis, whereas the elas- 
tic constant $K_{\mathrm{A}}$ is intrinsic to the overlayer. The angular width in the isotropic fluid may be approximated by

$$
G^{2}\left\langle\Delta \theta^{2}\right\rangle \sim\left(\frac{8 \pi}{3}\right)^{2} \frac{k_{\mathrm{B}} T}{h_{6} \xi_{6}^{2}}+\frac{G^{2} k_{\mathrm{B}} T}{4 \pi E_{\mathrm{c}} \xi_{\mathrm{p}}^{2}} \ln \frac{E_{\mathrm{c}}+h_{6}}{E_{\mathrm{c}}\left(\frac{\xi_{\mathrm{p}}}{\xi_{6}}\right)^{2}+h_{6}} .
$$

Here we have made the crude assumption that the Frank elastic constant jumps from 0 for $q<\xi_{6}^{-1}$ to $K_{\mathrm{A}}$ for $\xi_{6}^{-1}<q<\xi_{\mathrm{p}}^{-1}$. These bounds are accurate to factors of order $\pi$. If a hexatic transition occurs then $\xi_{6}$ is replaced by $\frac{2 \pi}{L}$, where $L$ is the sample size. At high temperatures

where one is well into the isotropic fluid phase, the first term in equation (4) should dominate. One might reasonably expect that $\xi_{6}$ will scale linearly with $\xi_{\mathrm{p}}$ away from the hexatic region so that $G \sqrt{\left(\Delta \theta^{2}\right)}$ will scale linearly with $\kappa$. As noted by Rosenbaum et al. [6], if $\xi_{6}$ diverges so that only the second term is left, then $G \sqrt{(\Delta \theta)^{2}}$ also scales linearly with $\kappa$. If $h_{6}$ and $E_{\mathrm{c}}$ were known accurately then it would be possible to estimate the relative weights of the two terms ; unfortunately this is not the case. Substituting plausible values into equation (4) leads one to the conclusion that the data are consistent with a hexatic phase but could also be explained by having the orientational correlation length $\xi_{6}$ at least 3 or 4 times the positional correlation length $\xi_{\mathrm{p}}$. At the minimum the orienting field $h_{6}$ must be sufficiently large to remove any remnant of the isotropic-hexatic transition. In brief, the single crystal data reported here strengthens the argument of Nagler et al. [6] that the orientational correlation length in the fluid phase is extraordinarily large if not infinite in the vicinity of the freezing transition.

Finally, as noted above, the solid xenon transverse and longitudinal scans are always broader than those for commensurate krypton. Further, as is evident in figures 3 and 4 , the deconvoluted profiles are elliptical with the radial width narrower then the transverse width. Thus the solid has the character of a " quenched hexatic ». Presumably, this occurs because the incommensurate system is more sensitive to impurities or surface defects. Surface impurities may or may not pin the local phase of the adsorbed xenon overlayer. In the former case they act as random fields which should destroy both the positional and the orientational long range order. In the latter case impurities act as pinning centres for dislocations, leading to a low temperature hexatic phase [13].

In summary, we have presented synchrotron X-ray data on the freezing of submonolayer xenon on single crystal graphite. The fluid positional correlation length evolves continuously up to at least $2000 \AA$ before the freezing transition occurs. Further, the excess orientational width scales linearly with the inverse positional correlation length $\kappa$, thus demonstrating that the orientational correlation length also becomes macroscopic either at or in advance of the freezing transition. These data are consistent with the dislocation mediated model of references [1] and [2], but they do not prove it uniquely. One important feature of the monolayer xenon solidification is that the transition is quite abrupt. Specifically, at $134 \mathrm{~K}$ the correlation length evolves from $10 \AA$ to $2000 \AA$ over a temperature range of $2 \mathrm{~K}$, or a reduced temperature range of $1.5 \%$. For the 2D $X Y$ model, the transition is much more gradual [14]. For the xenon on graphite system, all of the interactions are known accurately so that quantitative predictions based on the theories of reference [2] should be possible. Hopefully, these experiments will inspire such calculations in order to determine if the dislocation theories are indeed able to account quantitatively for this continuous two dimensional freezing transition. 


\section{Acknowledgments.}

These experiments were performed at the Stanford Synchrotron Radiation Laboratory which is supported by the U.S. Department of Energy, Office of Basic Energy Sciences. The work at MIT was supported by the U.S. National Science Foundation, Materials Research Laboratories under Contract No. DMR-81-19295, and by the U.S. Joint Services Electronics Program under Grant No. DAAG-29-83-K-0003. The work at Brookhaven was supported by the Division of Basic Energy Sciences, U.S. Department of Energy under Contract No. DE-ACO2-76CH00016. One of us (E.D.S.) was supported by an I.B.M. predoctoral fellowship, another (S.E.N.) by a Natural Sciences and Engineering Research Council of Canada postdoctoral fellowship.

\section{References}

[1] Kosterlitz, J. M. and Thouless, D. J., J. Phys. C 5 (1972) L124; 6 (1973) 1181 ; Berezinski, V. L., Sov. Phys. JETP 34 (1971) 34.

[2] Halperin, B. I. and Nelson, D. R., Phys. Rev. Lett. 41 (1978) 121 and 519 ; Nelson, D. R. and Halperin, B. I., Phys. Rev. B 19 (1979) 2457 ; Young, A. P., Phys. Rev. B 19 (1979) 1855.

[3] Chuі, S. T., Phys. Rev. Lett. 48 (1982) 935 ; Phys. Rev. B 28 (1983) 1978 ; Kleinert, H., Phys. Lett. A 95 (1983) 381.

[4] See for example, Moncton, D. E., Pindak, R., Davey, S. C. and Brown, G. S., Phys. Rev. Lett. 49 (1982) 1865.

[5] Heiney, P. A., Stephens, P. W., Birgeneau, R. J., Horn, P. M. and Moncton, D. E., Phys. Rev. $B 28$ (1983) 6416;

Dimon, P., Horn, P. M., Sutton, M., Birgeneau, R. J. and Moncton, D. E., Phys. Rev. B 31 (1985) 437.

[6] Nagler, S. E., Horn, P. M., Rosenbaum, T. F., Birgeneau, R. J., Sutton, M., Mochrie, S. G. J., Moncton, D. E. and Clarke, R., Phys. Rev. B (in press);

Rosenbaum, T. F., Nagler, S. E., Horn, P. M. and Clarke, R., Phys. Rev. Lett. 50 (1984) 1589 ; AépPli, G. and Bruinsma, R., Phys. Rev. Lett. 53 (1984) 2133.

[7] Specht, E. D., Sutton, M., Birgeneau, R. J., Moncton, D. E. and Horn, P. M., Phys. Rev. B 30 (1984) 1589.

[8] Abraham, F. F., Phys. Rev. B 29 (1984) 2906 ;

Hurlbut, S. B. and Dash, J. G., Phys. Rev. Lett. 53 (1984) 1931.

[9] D’Amico, K. L., Moncton, D. E., SPecht, E. D., Birgeneau, R. J., NAgler, S. E. and Horn, P. M., Phys. Rev. Lett. 53 (1984) 2250.

[10] Moncton, D. E. and Brown, G. S., Nucl. Instrum. Methods 208 (1983) 579.

[11] Tномy, A. and Duval, X., J. Chim. Phys. 66 (1969) 1966 ; 67 (1970) 2861 and 1101 ;

for a review see Thomy, A., Duval, X. and Regnier, J., Surface Sci. Rep. 1 (1981) 1.

[12] Higher quality graphite substrates with single crystal peaks as narrow as $0.01^{\circ} \mathrm{HWHM}$ have recently been obtained by one of us (K.D.) ; experiments on these substrates will more fully exploit the very high angular resolution of the synchrotron X-ray technique.

[13] Nelson, D. R., Phys. Rev. B 27 (1983) 2909;

Cardy, J. and Ostland, S., Phys. Rev. B 25 (1982) 6899.

[14] Heinekamp, S. and Pelcovits, R., private communication.

[15] Stephens, P. W., Heiney, P. A., Birgeneau, R. J., Horn, P. M., Moncton, D. E. and Brown, G. S., Phys. Rev. B 29 (1984) 3512. 